\title{
ALIGNING FUNCTIONS DEFINED ON CANTOR SETS
}

\author{
BY \\ JO FORD AND E. S. THOMAS, JR.(')
}

1. Introduction. In this paper we use the following notation and conventions. Euclidean $n$-space is denoted $R^{n}$ and, by the $n$-cube $I^{n}$ in $R^{n}$, we mean

$$
\left\{\left(x_{1}, \ldots, x_{n}\right) \mid 0 \leqq x_{i} \leqq 1\right\} \text {. }
$$

The $n$-sphere, $S^{n}$, is considered as the boundary of $I^{n+1}$. The combinatorial boundary and interior of a cell $B$ are denoted by $\partial B$ and int $B$ respectively. If $B$ is an $n$-cell lying in $I^{n}$ then the interior of $B$ relative to $I^{n}$ will be denoted: int $B$, rel $I^{n}$, or simply by: int $B$.

Frequently we shall consider $I^{n}$ as the product $I^{n-1} \times I$. If $p \in I$, then, to conserve space, we shall write $I^{n-1} \times p$ rather than $I^{n-1} \times\{p\}$. The symbol $\pi$ denotes the natural projection of $I^{n}$ onto $I^{n-1} \times 0$.

Let $f: K \rightarrow I^{n-1}$ be a function whose domain $K$ lies in $I^{n}$. We say that $f$ can be aligned provided there is a self-homeomorphism $u$ of $I^{n}$ such that $\pi u / K=f$, where $I^{n-1}$ has been identified in the natural way with $I^{n-1} \times 0$. Thus, $f$ can be aligned provided it has an extension $g$ mapping $I^{n}$ into $I^{n-1}$ such that $g$ is equivalent to $\pi$.

In [2] it was shown that if $K$ is a Cantor set in $I^{2}$ and $f: K \rightarrow I$ is a continuous map such that $f^{-1}(0)=K \cap(0 \times I)$ and $f^{-1}(1)=K \cap(1 \times I)$ then $f$ can be aligned. In this paper a corresponding result in higher dimensions is proved. The two main difficulties are that $K$ may hit $\partial I^{n}$ and that, for $n \geqq 3, K$ may not be nicely imbedded in $I^{n}$.

The latter possibility may be illustrated by letting $K$ be a Cantor set which lies in int $I^{n}$ whose complement in int $I^{n}$ is not simply connected. These exist for all $n \geqq 3$, [4]. Let $f$ map all of $K$ to a point in int $I^{n-1}$. Then $f$ cannot be aligned [4, Theorem 3E].

Thus, some sort of tameness condition on $K$ must be added. In $\S 2$ we define and investigate such a notion for Cantor sets in $I^{n}(n \geqq 3)$. In $\$ \$ 3,4$ and 5 we prove the main theorem of this paper, which is stated as Theorem 2 in $\S 3$.

In $\S 6$ we establish some miscellaneous results; a generalization of the main theorem, a proof of its converse and another characterization of tameness in $I^{n}$.

The authors thank R. Z. Goldstein and R. P. Osborne for helpful comments during the preparation of this paper.

2. Tameness in $I^{n}$. For completeness we begin with some well-known definitions and facts. An arc $\alpha$ in $R^{n}$ or $S^{n}$ is tame (in $R^{n}$ or $S^{n}$ ) provided there is an

Received by the editors April 3, 1968.

(1) Supported by NSF Grant GP-5935. 
ambient homeomorphism taking $\alpha$ onto a polyhedral arc and a Cantor set $K$ in $R^{n}$ or $S^{n}$ is tame provided it lies on a tame arc.

For Cantor sets in $I^{n}$, the following notion of tameness is introduced:

A Cantor set $K$ in $I^{n}$ is tame in $I^{n}$ if $K$ is tame in $R^{n}$ and $K \cap \partial I^{n}$ is tame in $\partial I^{n}$ (where $\partial I^{n}$ is identified with $S^{n-1}$ ).

Nontame Cantor sets exist in $R^{n}$ and $S^{n}$ for every $n$ except $n=1,2$; [1], [4]. In [3], Bing characterized tame Cantor sets in $R^{3}$ and recently Osborne extended this to all dimensions $\geqq 3$, as follows:

OSBORne's THEOREM [7]. A Cantor set $K \subset R^{n}$ is tame if and only if for each $\varepsilon>0$ there exists a collection of disjoint closed $n$-cells each of diameter less than $\varepsilon$ whose interiors cover $K$.

A collection of cells satisfying the above conditions will be called a "nice $\varepsilon$-cover of $K$ in $R^{n}$ ". The result also holds with $R^{n}$ replaced by $S^{n}$ and, as Osborne indicates in his proof, the $n$-cells can be required to be flat, as defined in [5].

Corresponding to this, if $K$ is a Cantor set in $I^{n}$ and $\varepsilon>0$, then a "nice $\varepsilon$-cover of $K$ in $I^{n}$ " will be a finite collection of disjoint closed $n$-cells lying in $I^{n}$ whose interiors, rel $I^{n}$, cover $K$ such that each cell has diameter less than $\varepsilon$ and each one intersecting $\partial I^{n}$ intersects it in an $(n-1)$-cell.

Osborne's theorem suggests the following:

THEOREM 1. A Cantor set $K \subset I^{n}$ is tame in $I^{n}$ if and only if for each $\varepsilon>0$ there is a nice $\varepsilon$-cover for $K$ in $I^{n}$.

Proof. Suppose $K$ is a Cantor set in $I^{n}$ such that for each $\varepsilon>0$ there is a nice $\varepsilon$-cover for $K$ in $I^{n}$. Suppose $\varepsilon>0$ and $C_{1}, \ldots, C_{m}$ is a nice $\varepsilon$-cover of $K$ in $I^{n}$. Each $C_{i}$ which meets $\partial I^{n}$ can be "thickened" slightly to obtain a nice $\varepsilon$-cover of $K$ in $R^{n}$. Hence, by Osborne's Theorem, $K$ is tame in $R^{n}$. Also the cover $C_{1}, \ldots, C_{m}$ immediately yields a nice $\varepsilon$-cover of $K \cap \partial I^{n}$ in $\partial I^{n}$. Hence by Osborne's Theorem for spheres, $K \cap \partial I^{n}$ is tame in $\partial I^{n}$. Hence $K$ is tame in $I^{n}$.

For the converse, suppose $K$ is tame in $I^{n}$ and $\varepsilon>0$. For convenience, denote $K \cap \partial I^{n}$ by $K_{0}$. Let $C_{1}, \ldots, C_{m}$ be a nice $\varepsilon$-cover of $K_{0}$ in $\partial I^{n}$ with $(n-1)$-cells. There exist disjoint $n$-cells $D_{1}^{\prime}, \ldots, D_{m}^{\prime}$ in $I^{n}$ each of diameter $<\varepsilon$ such that for each $i$, $D_{i}^{\prime} \cap \partial I^{n}=C_{i}$. Each $D_{i}^{\prime}$ will be replaced with a cell $D_{i}$ which has the additional property that $\partial D_{i}$ does not intersect $K$.

The construction of $D_{1}$ goes as follows. Since $\partial C_{1}$ does not intersect $K$ and since $K$ is tame in $R^{n}$, disjoint cells $B_{1}, B_{2}, \ldots, B_{s}$ can be chosen lying in int $I^{n}$ such that $\partial B_{i} \cap K=\varnothing, \partial D_{1}^{\prime} \cap K \subset \bigcup_{i=1}^{s}$ int $B_{i}$, the diameter of $D_{1}^{\prime}+\bigcup_{i=1}^{s} B_{i}$ is still less than $\varepsilon$, and each int $B_{i}$ intersects $D_{1}^{\prime}$.

For each $i, K \cap B_{i}=K \cap$ int $B_{i}$ is tame in int $B_{i}$, so there is a homeomorphism $u_{i}$ of $B_{i}$ which is the identity on $\partial B_{i}$ and moves $K \cap B_{i}$ into int $D_{1}^{\prime}$. Let $u$ be the identity off $\bigcup_{i=1}^{s} B_{i}$ and agree with $u_{i}$ on $B_{i}$. Then $u$ is a homeomorphism and $D_{1}$ $=u^{-1}\left(D_{1}^{\prime}\right)$ has diameter less than $\varepsilon$ and its boundary misses $K$. 
Having constructed $D_{2}, \ldots, D_{m}$ in a similar way, note that, if $U$ denotes $I^{n}$ $-\left(D_{1} \cup \cdots \cup D_{m}\right)$ then $K \cap U$ is compact. Since $K$ was assumed tame in $R^{n}$, it follows easily from Osborne's Theorem that there is a collection of closed $n$-cells $D_{m+1}, \ldots, D_{p}$ lying in $U$, each of diameter $<\varepsilon$, whose interiors cover $K \cap U$. Thus, $D_{1}, \ldots, D_{p}$ is a nice $\varepsilon$-cover of $K$ in $I^{n}$.

3. Aligning functions on $I^{n}$. This and the next section are devoted to proving the main result of this paper.

THEOREM 2. Let $K$ be a tame Cantor set in $I^{n}(n \geqq 3)$ and let $f: K \rightarrow I^{n-1}$ be a continuous function such that $f^{-1}\left(\partial I^{n-1}\right)=K \cap \partial I^{n}$. Then $f$ can be aligned.

In this section we align $f$ on the boundary of $I^{n}$ (and a little more). We start with the following

Proposition. Let $C$ be a Cantor set contained in $p \times I \subset S^{n} \times I(n \geqq 1)$ where $p=(0, \ldots, 0,1) \in S^{n}$. (Here for convenience we are considering $S^{n}$ as

$$
\left.\left\{\left(x_{1}, \ldots, x_{n+1}\right) \mid \sum x_{i}^{2}=1\right\} \cdot\right)
$$

Suppose that $C$ misses $S^{n} \times\{0,1\}$ and that $g: C \rightarrow S^{n}$ is continuous.

Then there is a self-homeomorphism $u$ of $S^{n} \times I$ which leaves $S^{n} \times 0$ and $S^{n} \times 1$ (setwise) fixed and such that $\pi u$ restricted to $C$ is $g$. (Here $\pi$ is the projection of $S^{n} \times I$ onto $S^{n} \times 0$ and $S^{n}$ has been identified with $S^{n} \times 0$.)

Proof. Roughly speaking, we are going to draw the graph of $g$ running vertically in $S^{n} \times I$ and choose a homeomorphism of $S^{n} \times I$ which moves points of $C$ onto corresponding points on the graph. We begin by extending $g$ to a map, also called $g$, of $p \times I$ into $S^{n}$. Define $g((p, 0))=g((p, 1))=(p, 0)$. It remains to extend $g$ on intervals of the form $p \times(a, b)$; briefly, this can be done by mapping $p \times(a, b)$ in the obvious way onto the geodesic joining $g(a)$ and $g(b)$. (If $g(a)$ and $g(b)$ are antipodal choose any geodesic.) The extension is continuous.

Partition $p \times I$ into subintervals $p \times\left[a_{i}, a_{i+1}\right], 0=a_{1}<a_{2}<\cdots<a_{n+1}=1$, which are short enough so that $g\left(p \times\left[a_{i}, a_{i+1}\right]\right)$ does not contain the point antipodal to $g\left(\left(p, a_{i}\right)\right)$.

Define $u_{1}$ on $S^{n} \times I$ as follows. Fix $(p, x) \in p \times\left[a_{1}, a_{2}\right]$. There is a unique "geodesic" rotation, denoted $r_{x}$, which takes $p$ onto $g(x)$. (Uniqueness follows from the shortness of $\left[a_{1}, a_{2}\right]$.) Put

$$
\begin{aligned}
& u_{1}(s, x)=\left(r_{x}(s), x\right) \quad \text { if } a_{1} \leqq x \leqq a_{2} \\
& =\left(r_{a_{2}}(s), x\right) \text { if } a_{2} \leqq x \leqq 1 .
\end{aligned}
$$

Then $u_{1}$ is a self-homeomorphism of $S^{n} \times I$, it is the identity on $S^{n} \times 0$ and aligns $g$ on $S^{n} \times\left[a_{1}, a_{2}\right]$, i.e., if $(p, x) \in p \times\left[a_{1}, a_{2}\right]$, then $\pi u_{1}((p, x))=\left(r_{x}(p), 0\right)=(g(x), 0)$.

Next one constructs $u_{2}$ which is the identity on $S^{n} \times\left[a_{1}, a_{2}\right]$ and aligns $g$ on $S^{n} \times\left[a_{2}, a_{3}\right]$, and the process is continued getting $u_{1}, \ldots, u_{n}$. The composition of these $u_{i}$ is the required homeomorphism. 
The main result of this section is

THEOREM 3. Assume the hypotheses of Theorem 2. Then there is a self-homeomorphism $u$ of $I^{n}$ such that $u(K)$ misses $I^{n-1} \times\{0,1\}$ and $\pi u=f$ on $K \cap \partial I^{n}$.

Proof. Let $C=K \cap \partial I^{n}$; since $C$ is tame in $\partial I^{n}$ there is a homeomorphism $u_{0}$ of $\partial I^{n}$ such that $u_{0}(C)$ lies in a straight line segment which misses $I^{n-1} \times\{0,1\}$. Extend $u_{0}$ to a self-homeomorphism $u_{1}$ of $I^{n}$.

Consider $\partial I^{n}-\left(\right.$ int $\left.I^{n-1} \times\{0,1\}\right)$ as $S^{n-2} \times I$ where $S^{n-2} \times 0$ and $S^{n-2} \times 1$ are identified with $I^{n-1} \times 0$ and $I^{n-1} \times 1$, respectively. We may assume $u_{1}(C)$ lies in $p \times I$ where $p=(0, \ldots, 0,1) \in S^{n-2}$.

Define $g: u_{1}(C) \rightarrow S^{n-2}$ by $g\left(u_{1}(c)\right)=f(c)$. Since $n-2 \geqq 1$, the proposition applies and we obtain a homeomorphism $u_{2}$ of $S^{n-2} \times I$ such that $\pi u_{2}=g$ on $u_{1}(C)$ and such that $g\left(S^{n-2} \times 0\right)=S^{n-2} \times 0$ and $g\left(S^{n-2} \times 1\right)=S^{n-2} \times 1$. Extend $u_{2}$ over all of $\partial I^{n}$ and then over $I^{n}$ getting a homeomorphism $u_{3}$. Then $u=u_{3} \circ u_{1}$ satisfies the conclusion of Theorem 3.

4. Cell collections in $I^{n}$ and $I^{n-1} \times 0$. By virtue of Theorem 3, we may assume, for the remainder of the proof of Theorem 2 that $K$ misses the top and bottom faces of $I^{n}$ and that $f$ is already aligned on $\partial I^{n}$. The rest of the proof consists of pushing the points of $K \cap$ int $I^{n}$ over their images in $I^{n-1} \times 0$. This is accomplished by a sequence of successively smaller pushes, i.e., by a sequence of homeomorphisms which converge to a homeomorphism.

This section is devoted to constructing some collections of cells which determine what part of int $I^{n}$ to push and where to push it.

For each positive integer $i$, let $\mathscr{C}_{i}$ be a nice $2^{-(i+1)}$-cover of $K$ in $I^{n}$ such that:

(1) No member of any $\mathscr{C}_{i}$ meets $I^{n-1} \times\{0,1\}$;

(2) If $C \in \mathscr{C}_{i}$ then $f(K \cap C)$ has diameter $<2^{-(i+2)}$ and

(3) $\mathscr{C}_{i+1}$ strongly refines $\mathscr{C}_{i}$, i.e., if $C \in \mathscr{C}_{i+1}$ then there is a (unique) $C^{\prime} \in \mathscr{C}_{i}$ such that $C \subset$ int $C^{\prime}\left(\operatorname{rel} I^{n}\right)$.

It is useful to split up $\mathscr{C}_{i}$ as follows:

$$
\mathscr{E}_{i}=\left\{C \in \mathscr{C}_{i} \mid C \cap \partial I^{n} \neq \varnothing\right\}, \quad \mathscr{I}_{i}=\left\{C \in \mathscr{C}_{i} \mid C \cap \partial I^{n}=\varnothing\right\} .
$$

These are called the end cells and interior cells of $\mathscr{C}_{i}$, respectively.

We now choose collections of cells in $I^{n-1} \times 0$ which will be used to align the elements of the $\mathscr{C}_{i}$. These collections $\left\{D(C) \mid C \in \mathscr{I}_{i}\right\}$ and $\left\{D^{\prime}(C) \mid C \in \mathscr{I}_{i}\right\}$ are to have the following properties:

(1) Each $D(C)$ and $D^{\prime}(C)$ is a closed $(n-1)$-cell in the interior of $I^{n-1} \times 0$ having diameter $<2^{-(i+1)}$.

(2) For each $C, f(C \cap K) \subset$ int $D(C)$ and $D(C) \subset$ int $D^{\prime}(C)$.

(3) If $C_{1} \in \mathscr{I}_{i}, C_{2} \in \mathscr{I}_{j}(i<j)$ and $C_{2} \subset C_{1}$, then $D^{\prime}\left(C_{2}\right) \subset$ int $D\left(C_{1}\right)$.

Their construction depends upon the fact that if $U$ is a closed subset of diameter less than $\varepsilon$ in the interior of the convex $(n-1)$-cell $H$ in $I^{n-1} \times 0$, then there are two convex $(n-1)$-cells, $U_{3}$ and $U_{4}$, of diameter less than $2 \varepsilon$ such that $U \subset \operatorname{int} U_{3}$, 
$U_{3} \subset \operatorname{int} U_{4}$, and $U_{4} \subset$ int $H$. The construction is inductive, using $U$ to be some $f(C \cap K)$ and $H$ to be either $I^{n-1} \times 0$ or a previously constructed $D(C)$.

5. The sequence of homeomorphisms. The proof is now completed by constructing a sequence $u_{1}, u_{2}, \ldots$ of self-homeomorphisms of $I^{n}$ with the following properties:

(A) If $x \in I^{n}$ then $d\left(u_{i}(x), u_{i+1}(x)\right)<2^{-i}$,

(B) If $x \in I^{n}-K$ then there exists $i$ such that $u_{i}(x)=u_{i+1}(x)=\cdots$,

(C) Each $u_{i}$ is the identity on $\partial I^{n}$ and there is an increasing sequence $i_{1}, i_{2}, i_{3}, \ldots$ of positive integers such that if $C \in \mathscr{I}_{i,}$, then there is a $k$ such that $u_{k}(C)=u_{k+1}(C)$ $=\cdots$, and

(D) If $k \in K$ then $d\left(\pi u_{i}(k), f(k)\right) \rightarrow 0$ as $i \rightarrow \infty$.

By condition (A), lim $u_{i}$ is a continuous function, $u: I^{n} \rightarrow I^{n}$. Conditions (B) and (C) imply that $u$ is a self-homeomorphism of $I^{n}$ and condition (D) implies that $u$ aligns $f$.

The construction is carried out inductively. To keep an already formidable amount of notation within reasonable limits we shall merely set up the machinery required for the general construction and produce $u_{1}$ and $u_{2}$. The inductive step is done in a similar way.

Choose an integer $i_{1}>1$ such that if $E \in \mathscr{E}_{i_{1}}$ and $E \subset E^{\prime} \in \mathscr{E}_{1}$, then $f(K \cap E)$ is interior to $\pi\left(E^{\prime}\right)$ in $I^{n-1}$.

If $D$ is a cell in $I^{n-1} \times 0$, then a cell in $I^{n}$ of the form $D \times J$ where $J$ is a closed interval in $(0,1)$ is called a pancake cell over $D$. For each $F \in \mathscr{I}_{i_{1}}$ choose pancake cells $\tilde{F}$ and $\tilde{F}^{\prime}$ over $D(F)$ and $D^{\prime}(F)$, respectively, of diameter less than $\frac{1}{4}$ such that $\tilde{F}$ is in the interior of $\tilde{F}^{\prime}$ and so that the $\tilde{F}^{\prime}$ are disjoint from each other and from the elements of $\mathscr{E}_{1}$.

Denote by $u_{1}$ a self-homeomorphism of $I^{n}$ which is the identity on $\partial I^{n}$ and each term of $\mathscr{E}_{i_{1}}$ and which has the property that, for each $F \in \mathscr{I}_{i_{1}}, \tilde{F} \subset \operatorname{int} u_{1}(F)$ and $u_{1}(F) \subset$ int $\widetilde{F}^{\prime}$, i.e., $u_{1}(F)$ lies between $\tilde{F}$ and $\tilde{F}^{\prime}$. Furthermore, $u_{1}$ can be constructed so that for each $E^{\prime} \in \mathscr{E}_{1}$, if $u_{1}(x) \in E^{\prime}$ then $x \in E^{\prime}$.

Note for each $k \in K$ the distance from $f(k)$ to $\pi u_{1}(k)$ is less than $\frac{1}{2}$.

We now construct $u_{2}$ by moving $u_{1}(K)$ around inside the cells of $\mathscr{E}_{1}$ and the cells $u_{1}(F)$ for $F \in \mathscr{I}_{i_{1}}$.

Choose $j>i_{1}$ so $m \geqq j$, and $E^{\prime \prime} \in \mathscr{E}_{m}, E^{\prime \prime} \subset E \in \mathscr{E}_{i_{1}}$ implies $f\left(K \cap E^{\prime \prime}\right) \subset \operatorname{int} \pi(E)$.

If $E$ is one such term of $\mathscr{E}_{i_{1}}$ and $E^{\prime}$ is the term of $\mathscr{E}_{1}$ containing it, then since $f(K \cap E) \subset$ int $\pi\left(E^{\prime}\right)$ it follows that for each point $x$ of $f(K \cap E)$ there is an $\varepsilon_{x}>0$ and a pancake cell over the $\varepsilon_{x}$-neighborhood of $x$ that lies in int $E^{\prime}$ and misses each term of $\mathscr{E}_{i_{1}}$. Using a finite covering of $f(K \cap E)$ there is a $k \geqq j$ such that $2^{-(k+2)}$ is less than each $\varepsilon_{x}$ used in the cover. Thus for each $F$ in $\mathscr{I}_{k}$ that is contained in $E$ there is a pancake cell over $D^{\prime}(F)$ interior to $E^{\prime}$ that misses each term of $\mathscr{E}_{i_{1}}$. Let $k=i_{2}$.

Pancake cells $\tilde{F}$ and $\tilde{F}^{\prime}$ over $D(F)$ and $D^{\prime}(F)$, respectively, of diameter $<\frac{1}{8}$ can be 
constructed for each $F$ in $\mathscr{I}_{i_{2}}$ such that $\tilde{F}$ is interior to $\tilde{F}^{\prime}$, and $\tilde{F}^{\prime}$ is interior to $E^{\prime} \in \mathscr{E}_{1}$ if $F=u_{1}(F) \subset E^{\prime}$ or $\tilde{F}^{\prime}$ is interior to the pancake cell $\tilde{G}$ (for some $G \in \mathscr{I}_{i_{1}}$ ) if $u_{1}(F) \subset \tilde{G}^{\prime}$. Furthermore, these pancake cells may be chosen so that the $\tilde{F}^{\prime}$ are disjoint and miss each term of $\mathscr{E}_{i_{1}}$.

It then follows that there is a self-homeomorphism $w_{2}$ of $I^{n}$ which is the identity on $\partial I^{n}$, each term of $\mathscr{E}_{i_{2}}$ and the complement of $\bigcup \mathscr{E}_{1}+\bigcup\left\{u_{1}(F) \mid F \in \mathscr{I}_{i_{1}}\right\}$, which takes each $u_{1}(F)$ for $F \in \mathscr{I}_{i_{2}}$ between $\tilde{F}$ and $\tilde{F}^{\prime}$, and which has the property that for each $E$ in $\mathscr{E}_{i_{1}}$ if $w_{2}(x)$ is in $E$ then $x$ is in $E$.

Letting $u_{2}=w_{2} u_{1}$ we note that

(a) $d\left(u_{1}(x), u_{2}(x)\right)<1 / 2$ for each $x \in I^{n}$,

(b) if $x \notin \bigcup\left\{F \mid F \in \mathscr{I}_{1}\right\}+\bigcup \mathscr{E}_{i_{0}}$ then $u_{2}(x)=u_{1}(x)$,

(c) if $C \in \mathscr{I}_{i_{1}}$ then $u_{1}(C)=u_{2}(C)$, and

(d) if $k \in K$, then $d\left(f(k), \pi u_{2}(k)\right)<1 / 4$.

The induction is carried out in the obvious way. In the "limit", conditions (a)-(d) become conditions (A)-(D) which were stated at the end of section 4 . Roughly speaking, conditions (1)-(4) and the conditions on the $\tilde{F}$ and $\tilde{F}^{\prime}$ guarantee that the next homeomorphism will not have to move points of $\mathscr{C}_{i}$, very far.

This completes the proof of Theorem 2.

6. Further remarks. Given $n \geqq 3$, and $m<n$ let $\pi_{m}: I^{n} \rightarrow I^{n}$ be given by

$$
\pi_{m}\left(x_{1}, \ldots, x_{n}\right)=\left(x_{1}, \ldots, x_{m}, 0, \ldots, 0\right) \in I^{n} .
$$

Thus $\pi_{n-1}$ is our $\pi$. One then has the following generalization:

THEOREM 4. If $K$ is a tame Cantor set in $I^{n}(n \geqq 3)$ and $f: K \rightarrow I^{m}(m<n)$ is a continuous map such that $f^{-1}\left(\partial I^{m}\right)=K \cap \partial I^{n}$ then there exists a self-homeomorphism $u$ of $I^{n}$ such that $\pi_{m} u=f$ on points of $K$.

To see this, let $i_{m}: I^{m} \rightarrow I^{n-1}$ be given by

$$
i_{m}\left(\left(x_{1}, \ldots, x_{m}\right)\right)=\left(x_{1}, \ldots, x_{m}, 1 / 2, \ldots, 1 / 2\right) \in I^{n-1} .
$$

Then the map $i_{m} f: K \rightarrow I^{n-1}$ satisfies the boundary condition of Theorem 2 . Choose a $u$ which aligns $i_{m} f$; then, also, for each $k \in K, \pi_{m} u(k)=f(k)$ as required.

The next result says that the definition of tame in $I^{n}$ which we have selected is the right one for our purposes.

THEOREM 5. Let $K$ be a Cantor set in $I^{n}(n \geqq 3)$ such that every continuous function from $K$ into $I^{n-1}$ can be aligned; then $K$ is tame in $I^{n}$.

Proof. Let $f: K \rightarrow I^{n-1}$ be a continuous function such that $f(K)$ lies in

$$
\left\{\left(x_{1}, \ldots, x_{n-1}\right) \mid x_{1}=1 / 2\right\},
$$

$f^{-1}\left(\partial I^{n-1}\right)=K \cap \partial I^{n}$ and $f\left(K \cap \partial I^{n}\right)=(1 / 2,0, \ldots, 0)=p \in \partial I^{n-1}$. Let $u$ be a homeomorphism of $I^{n}$ which aligns $f$. Then the restriction of $u$ to $\partial I^{n}$ moves $K \cap \partial I^{n}$ into the tame arc $p \times I$ hence $K \cap \partial I^{n}$ is tame in $\partial I^{n}$. Extend $u$ to a selfhomeomorphism $w$ of $R^{n}$. Then $w$ moves $K$ into the hyperplane $x_{1}=1 / 2$ in $R^{n}$. As is 
well known, a result of Klee [6, p. 36] implies that $w(K)$, and hence $K$ itself, is tame in $R^{n}$.

By slightly modifying the proof of the last result we obtain the following characterization of tameness in $I^{n}$.

THeOREM 6. A Cantor set $K \subset I^{n}(n \geqq 3)$ is tame in $I^{n}$ if and only if there is a homeomorphism of $I^{n}$ taking $K$ into $H_{1 / 2}=\left\{\left(x_{1}, \ldots, x_{n}\right) \mid x_{1}=1 / 2\right\}$.

Proof. The necessity of the condition is proved by aligning a suitably chosen function from $K$ into $I^{n-1}$. To prove sufficiency suppose $u$ satisfies the above condition. By the last part of the argument for Theorem $5, K$ is tame in $R^{n}$. It remains to prove that $K \cap \partial I^{n}$, denoted $C$, is tame in $\partial I^{n}$. Choose a homeomorphism $w$ of $\partial I^{n}$ which leaves some point $p \in H_{1 / 2} \cap \partial I^{n}$ fixed and agrees with $u$ on $C$. We identify $\partial I^{n}-\{p\}$ with $R^{n-1}$ and $\left(H_{1 / 2} \cap \partial I^{n}\right)-\{p\}$ with $R^{n-2} \subset R^{n-1}$. Then $w /\left(\partial I^{n}-\{p\}\right)$ is a homeomorphism of $R^{n-1}$ which takes $C$ into $R^{n-2}$. Thus, by [6] again, $C$ is tame in $R^{n-1}$. This implies that $K \cap \partial I^{n}$ is, after all, tame in $\partial I^{n}$.

As a result of Theorem 6 we have the following theorem. Its converse is false since a wild Cantor set in $\partial I^{n}$ is tame in $R^{n}$.

TheOREm 7. Suppose $K$ is a Cantor set in $I^{n}$. If $K$ is tame in $I^{n}$ then there is an arc $\alpha$ containing $K$ and contained in $I^{n}$ such that $\alpha$ is tame (in $\left.R^{n}\right)$.

Proof. The theorem is clearly true if $n=2$ so suppose $n \geqq 3$. Then, by Theorem 6 there is a self-homeomorphism $u$ of $I^{n}$ such that $u(K)$ lies in an $(n-1)$-hyperplane, $H$. But $I^{n} \cap H$ contains an arc $\alpha$ which contains $u(K)$ and since $\alpha$ lies in the $(n-1)$ hyperplane $H$ it is tame in $R^{n}$. Thus $u^{-1}(\alpha)$ is tame in $R^{n}$ and $K \subset U^{-1}(\alpha) \subset I^{n}$.

\section{BIBLIOGRAPHY}

1. L. Antoine, Sur l'homéomorphisme de deux figures et de leurs voisinages, J. Math. Pures Appl. (8) 4 (1921), 221-325.

2. B. J. Ball, Jo Ford and E. S. Thomas, Jr., Extending functions defined on a subset of a disk, Proc. Amer. Math. Soc. 20 (1969), 75-80.

3. R. I. Bing, Tame Cantor sets in $E^{3}$, Pacific J. Math. 11 (1961), 435-446.

4. W. A. Blankinship, Generalization of a construction of Antoine, Ann. of Math. (2) 53 (1951), 276-297.

5. M. Brown, Locally flat imbeddings of topological manifolds, Ann. of Math. (2) 75 (1962), 331-341.

6. V. L. Klee, Some topological properties of convex sets, Trans. Amer. Math. Soc. 78 (1955), $30-45$.

7. R. P. Osborne, Embedding Cantor sets in a manifold, Michigan Math. J. 13 (1966), 57-63.

AUBURN UNIVERSITY, Auburn, Alabama

UNIVERSITY OF MiCHIGAN, ANn Arbor, Michigan 